\title{
Study of flora of Miandasht Wildlife Refuge in Northern Khorassan Province, Iran (a)
}

\author{
Rahimi A. ${ }^{1 \star}$ and Atri M. ${ }^{2}$ \\ ${ }^{1}$ Department of Biology, Bojnourd Branch, Islamic Azad University, Bojnourd, Iran. \\ ${ }^{2}$ Department of Biology, Bu Ali-Sina University, Hamedan, Iran.
}

Accepted 5 August, 2013

\begin{abstract}
A wide area of Iran is covered by arid and semiarid regions. In this survey, flora of an area of the Miandasht Wildlife Refuge, out of the safe part, was studied. This region covers $84435 \mathrm{Ha}$, situated in the west of Khorassan province in Iran. The climate of the area according to de Martone system is semiarid. The mean annual precipitation is $275 \mathrm{~mm}$ and the altitude varies from 931 to $1021 \mathrm{~m}$ above sea level. Plants were collected from 2008 to 2011. A total of 256 taxa belonging to 152 genera and 35 families from Angiospermae and Gymnospermae were found. Asteraceae, Chenopodiaceae, Brassicaceae and Fabaceae were the greatest families, respectively. Geraniaceae, Ixioliriaceae, Orobanchaceae, Plantaginaceae, Primulaceae, Resedaceae and Rosaceae, each included one species. Based on Raunkiaer life form classification system, majority of the species $(55.86 \%)$ were therophytes. Other life forms in descending order were hemicryptophytes (15.62\%), chamaephytes (10.16\%), phanerophytes (8.6\%) and geophytes (9.38\%). Chorologicaly, most of the species were Irano-Turanian. Flora of Miandasht Wildlife Refuge include 20 low risk species and 29 (11.6\%) endemic of Iran species. 67 pasture species and 38 medicinal species were distinguished. Most of the species were invasive plants. They are established in this area because of overgrazing and natural disturbance.
\end{abstract}

Key words: Flora, Miandasht Wildlife Refuge, Iran endemic, life form, chorotype.

\section{INTRODUCTION}

Iran in terms of topography, climate, vegetation and geographical features is one of the most important and unique countries in the Middle East. According to a recent study (Mozaffarian, 2007), flora of Iran includes 8000 species belonging to 1450 genera and 150 families. These families include 124 dicotyledons, 22 monocotyledons and 4 gymnosperms.

Some resources related to vegetation of Iran are as follows: Flora Orientalis (Boissier, 1936), Flora Keredjensis (Bornmuller and Gauba, 1935-1940), Flore de l'Iran (Parsa, 1948-1952), Flora Iranica (Rechinger, 1963-2005) Flora of Iran, Tracheophyta (Mobayen, 1975-1995), Colored Flora of Iran (Ghahreman, 1977-2007) and Flora of Iran (Assadi et al., 1988-2011).
One of the most extensive areas for speciation in holarctic kingdom is located in Iran (Akhani, 2006). Also, some studies in the field of semi deserts and deserts of Iran have been conducted. For instance, studies on the autumn plants of Kavir, Iran (Assadi, 1984), plants of the Kavir Protected Area, Iran (Rechinger and Wendelbo, 1976), Plants of the Touran Protected Area, Iran (Rechinger, 1977), Notes on the distribution, climate and flora of the sand deserts of Iran and Afghanistan (Freitag, 1986).

Some case studies have been performed in deserts of Iran, for example: A contribution to the vegetation and flora of Kavire Meyghan, Iran (Akhani, 1989), floristic and cartographic study of protected area of Ghamishloo

*Corresponding author. E-mail: noosha2@yahoo.com. Tel: (009821) 2208916. 
(Yousofinajafabadi, 1996), study of the flora of the Kabar dam of Ghom (Tavakkoli and Mozaffarian, 2005) and flora of halophytes in Iran (Asri, 2007).

A fundamental role of government conservation agencies is to set priorities for the conservation and management of biodiversity (David and Kenneth, 2001). To evaluate the status of biodiversity and to determine how current conservation efforts can be improved, biodiversity monitoring is crucial (Kerstin et al., 2013). The nature and quality of vegetation cover is an important factor for soil conservation through its role in reducing the erosive impact of precipitation degraded areas in semi-arid regions (Turan and Filiz, 2011).

The objective of this study was to provide urgently needed scientific support for programs of biodiversity conservation. The Miandasht Wildlife Refuge (MWR) covers $84435 \mathrm{Ha}$, and is located in North Khorassan province $\left(56^{\circ}, 26^{\prime}\right.$ to $56^{\circ}, 57^{\prime}$ longitude and $47^{\circ}, 30^{\prime}, 36^{\prime \prime}$ to $37^{\circ}, 30^{\prime}$ latitude) Figure 1 . The mean annual precipitation is $250 \mathrm{~mm}$ and the altitude varies from 912 to $1085 \mathrm{~m}$ above sea level. The climate is semiarid and chorologicaly located in the Irano-Turanian region. A wide area of MWR is rangeland. Early and uncontrolled grazing in these rangelands led to the decrease of the production by pastured plants, imbalances in the ecosystem, disruption in water and food cycles, increasing the unfavorable species and decreasing the quantity of vegetation and soil erosion. Also, a wide part of this region is covered by sand and saline soils. Totally, approximately 40 to $50 \%$ of critical points of erosion around of Jajarm city is located in this area, and the plants are permanently exposed to environmental stresses. MWR is habitat for important animal species, such as: the Asiatic cheetah which is rare worldwide and its survival is one of the most important goals of the Environmental Protection Agency, so the study of various environmental aspects of this area is essential for the balance and stability of the ecosystem, particularly vegetation which is the first loop of the animal food chains.

Aims of this study were to introduce the flora of MWR, to detect endemism, vulnerability and chorology of species, and to distinguish medicinal and pasture plants in $12000 \mathrm{Ha}$ out of the safe part of the MWR. This study is intended as a useful tool for policy markers and scientists to advocate for modifications in national legislation and policy aimed at conservation and combating desertification. Analyzing species richness, extinction level and distribution drivers are important preliminary steps to set conservation priorities and to test environmental policies (Giuseppe, 2013).

In order to determine the influence of protection from grazing on diversity of plant species, flora of the safe part was studied which will be written in another article.

\section{MATERIALS AND METHODS}

Basic information on MWR was obtained using geological and topographical maps $1 / 50000$ and aerial photos of this area. Then, by scrolling in the area, its boundaries were determined using handheld Garmin's GPS map76CS. All plants were photographed by means of a digital camera (Nikon D70S). Plants were identified using stereomicroscope, keys and descriptions in available scientific resources, specially flora Iranica (Rechinger, 1963-2005), Flora of Iran (Assadi et al., 1988-2011) and flora of the adjacent countries, namely flora of Turkey and the East Aegean Island (Davis, 1965-1985). Flora of U.S.S.R (Komarov, 1934-1957), Flora of West Pakistan (Nasir and Qaser, 1970-2001) and Flora of Iraq (Townsend and Guest, 1966-1986).

Finally, names of all plants were confirmed by taxonomists in the research institute of forests and rangelands of Iran. The life forms of species were distinguished according to the life form classification (Raunkiaer, 1934). The geographical distribution of each species was assessed from reviews, monographs and distributional data in the floras, particularly Flora Iranica (Rechinger, 1963-2005). The terminology and delimitation of the main phytogeographic areas, that is, Euro-Siberian (ES), Irano-Turanian (IT), Mediterranean (M) and within Euro-Siberian region relate to standard works of reference, particularly those of Zohary (1973), Takhtajan(1986) and flora Iranica (Rechinger, 1963-2005). The IUCN Red List Categories (Jalili and Jamzad, 1999) are used to designate the threat categories.

Pasture plants were detected by asking the villagers by direct observations, and by using the Codes of Pasture Plants (Publication Committee and Propaganda of the Research Institute of Forestsand Rangelands of Iran 1982).

Medicinal plants were determined using the available scientific resources, for example: Amin (1991), Zargary (1999) and Javidtalesh (2001).

\section{RESULTS AND DISCUSSION}

Totally, 256 taxa belonging to 152 genera and 35 families of Angiospermae and Gymnospermae were determined in the study area (Table 1, Figure 5-12). These families consist of 1 Gymnospermae, 29 Dicotyledons and 5 Monocotyledons.

According to Table 1, the following families had the highest number of species: $43(16.8 \%)$ Asteraceae, 41 (16.02\%) Chenopodiaceae, 32 (12.5\%) Brassicaceae and 22 (8.59\%) Fabaceae (Figure 2). These results are consistent with the results of most studies in similar areas (Rechinger and Wendelbo, 1977; Asri, 2003; Yousofinajafabadi, 1996).

Presumably uncontrolled grazing caused the maximum number of Asteraceae species (Tavakkoli and Mozaffarian, 2005). Since the studied area is at risk due to early and excessive grazing, the maximum number of Asteraceae species is justifiable. In the study of halophytes of Iran (Asri, 2007), the numbers of Chenopodiaceae species were large. The large number of this taxon species can be explained by saline soils which are widely spread in this region.

The large number of Chenopodiaceae, Asteraceae and Brassicaceae are indicator of desert conditions (Saberamoli et al., 2001). Table 1 illustrates that the genera of Astragalus, Salsola, Atriplex and Valerianella have the highest number of species with 17, 9, 6 and 6 species, respectively. Irano-Turanian region is the major origin of Astragalus, and $91 \%$ of Astragalus species Iran, grow in 
Table 1. List of plants in the Miandasht Wildlife Refuge (out of the safe part) and some of their features.

\begin{tabular}{|c|c|c|c|c|c|c|}
\hline Scientific name of taxon & L.F & Chor & $\mathrm{Pa}$ & Me & En & $\mathrm{Vu}$ \\
\hline \multicolumn{7}{|l|}{ Apiaceae } \\
\hline Cuminum setifolium L. & Th & IT & & * & & \\
\hline Dorema aitchisonii Korov. ex M. Pimen. & Hem & IT & & & & \\
\hline Ducrosia anetifolia (DC.) Boiss. & Hem & IT-SS & * & * & & \\
\hline Eryngium bungei Boiss. & Hem & $\mathrm{IT}$ & * & & & \\
\hline Psammogeton canescens (DC.) V. & Th & IT & & & & LR \\
\hline Schumannia karelinii (Bunge) Korov. & G.t & IT-SS & & & & \\
\hline \multicolumn{7}{|l|}{ Asteraceae } \\
\hline Acantholepis orientalis Less. & Th & IT & * & & & \\
\hline Acroptilon repens (L.) DC. & Hem & IT & & * & & \\
\hline Amberboa turanica Lljin & Th & IT & & & & \\
\hline Amberboa nana (Boiss.) Lljin. & Th & IT & & & & \\
\hline Anthemis austero-iranica Rech. f., Aell. \& Esfand. & Th & IT-SS & & & * & LR \\
\hline Anthemis rhodocentra Iranshahr & Th & IT-SS & & & & \\
\hline Artemisia scoparia Waldst. \& Kit. & $\mathrm{Ch}$ & IT-ES & * & * & & \\
\hline Artemisia sieberi Besser. & $\mathrm{Ch}$ & IT & * & * & & \\
\hline Carthamus oxyacantha M. B. & Th & IT-M-SS & & & & \\
\hline Centaurea bruguieriana (DC.)Hand. & Th & IT & & & & \\
\hline Centaurea pulchella Ledeb. & Th & IT & & & & \\
\hline Cousinia lasiandra Bunge. & Hem & IT & & & * & LR \\
\hline Cousinia neurocentra Bunge. & Hem & IT & & & * & LR \\
\hline Cousinia piptocephala Bunge. & Hem & IT & & & * & LR \\
\hline Cousinia prolifera Jaub. \& Spach. & Th & IT & & & & \\
\hline Cousinia turkmenorum Bornm & Th & IT & & & & \\
\hline Crepis sancta (L.) Babcock. & Th & IT-M & & & & \\
\hline Dipterocome pusilla Fisch \& C. A. Mey. & Th & IT & & & & \\
\hline Echinops leucographus Bunge & Hem & IT & & & & \\
\hline Echinops pungens Trautv. & Hem & IT & & & & \\
\hline Epilasia acrolasia (Bunge.) C. B. Clarke. & Th & IT & & & & \\
\hline Epilasia hemilasia (Bunge.) C. B. Clarke. & Th & IT & & & & \\
\hline Filago arenaria $\mathrm{L}$. & Th & IT & & & & \\
\hline Gymnarrhena micrantha Desf. & Th & IT-SS & & & & \\
\hline Heteroderis pusilla (Bunge) Boiss. & Th & $\mathrm{IT}$ & & & & \\
\hline Koelpinia linearis Pall. & Th & IT-SS & & & & \\
\hline Koelpinia tenuissima Pavl. \& Lipsch & Th & IT & & & & \\
\hline Lactuca serriola L. & Hem & $\mathrm{IT}$ & * & * & & \\
\hline Lasiopogon muscoides (Desf.) DC. & Th & IT-SS & & & & \\
\hline Launaea acanthodes (Boiss.) & Hem & $\mathrm{IT}$ & & * & DD & \\
\hline \multicolumn{7}{|l|}{ Mausolea eriocarpa (Bge.) Poljak. ex Podl. } \\
\hline Microcephala lamellata (Bunge) Pobed. & Th & IT & & & & \\
\hline Oligochaeta minima (DC.) C. koch. & Th & $\mathrm{IT}$ & & & & \\
\hline Pulicaria gnaphalodes (Vent.) Boiss. & Hem & IT-SS & & & & \\
\hline Senecio glaucus $\mathrm{L}$. & Th & IT-M-SS & & & & \\
\hline Scariola orientalis (Boiss.) Sojak. & Hem & IT & & & & \\
\hline Scorzonera lituisinowa Fisch. \& C.A.Mey. & G.t & IT & & & & \\
\hline Scorzonera paradoxa Fisch. \& C.A. Mey. & G.t & IT & & & & \\
\hline Scorzonera pusilla Pall. & G. t & IT & & & & \\
\hline Scorzonera raddeana C. Winkl. & G. $t$ & IT & & & & \\
\hline Scorzonera rigida Auch. & G. $t$ & IT & & & & \\
\hline Thevenotia persica DC. & Th & IT & & & & \\
\hline
\end{tabular}


Table 1. Contd.

\begin{tabular}{|c|c|c|c|c|c|c|}
\hline Xanthium stromarium $\mathrm{L}$. & Th & IT & & * & & \\
\hline \multicolumn{7}{|l|}{ Boraginaceae } \\
\hline Arnebia decumbens (Vent.) Coss. \& Kral. & Th & IT-SS & * & & & \\
\hline Arnebia linearifolia DC. & Th & IT-SS & & & & \\
\hline Gastrocotyle hispida (Forssk.) C. B. Clarke & Th & IT-SS & & & & \\
\hline Heliotropium aucheri DC. & Hem & IT & & & & \\
\hline Heliotropium europaeum $\mathrm{L}$. & Th & IT -ES & * & * & & \\
\hline Heliotropium dasycarpum Ledeb. & Hem & IT & & & & \\
\hline Heterocaryum subsessile Vatke, Zeitschr. Gesammt. & Th & IT-SS & & & & \\
\hline Lappula ceratophora (M. Pop.) M. Pop. & Th & IT & & & & \\
\hline Lappula semiglabra (Ledeb.) Gurke & Th & IT & & & & \\
\hline Lappula sesiliflora (Boiss.) Gurke & Th & IT & & & & \\
\hline Lappula spinocarpus (Forssk.) Ascherson \& O. Kuntze & Th & IT-SS & & & & \\
\hline Microparacary bungei (Boiss.) Khatamsaz, comb .Nov. & Th & IT-SS & & & & \\
\hline Nonnea caspica (Willd.) G. Don & Th & IT & & & & \\
\hline \multicolumn{7}{|l|}{ Brassicaceae } \\
\hline Aethionema carneum (Banks \& Soland.) B. Fedtsch. & Th & IT & & & & \\
\hline Alyssum dasycarpum Steph. ex Willd. & Th & IT & * & * & & \\
\hline Alyssum linifolium Steph. ex Willd. & Th & IT-ES-M & * & * & & \\
\hline Alyssum marginatum Steud. ex Boiss. & Th & IT & & * & & \\
\hline Arabidopsis pumila (Steph.) N. Bosch. & Th & IT & & & & \\
\hline Cardaria draba (L.) Desv. & G. $r$ & IT & * & * & & \\
\hline Cryptospora falcata Kar. \& Kir. & Th & IT & & & & \\
\hline Descurainia sophia Webb. \& Berth. & Th & IT-M-ES & * & * & & \\
\hline Erysimum crassicaule (Boiss.) Boiss. & Hem & IT & * & & * & LR \\
\hline Euclidium syriacum (L.) R. Br. & Th & IT & * & & & \\
\hline Goldbachia laevigata DC. & Th & IT & * & * & & \\
\hline Goldbachia verrucosa DC. & Th & IT & & & & \\
\hline Isatis buschiana Schischk. & Hem & IT & & & & \\
\hline Isatis emarginata Kar. \& Kir. & Th & IT & & & & \\
\hline Isatis minima Bge. & Th & IT & & & & \\
\hline Lepidium perfoliatum $\mathrm{L}$. & Th & IT-M-ES & * & & & \\
\hline Lepidium vesicarium $\mathrm{L}$. & Th & IT & & & & \\
\hline Leptaleum filifolium (Willd.) DC. & Th & IT & * & & & \\
\hline Malcolmia africana (L.) R. Br. & Th & IT-M-SS & * & & & \\
\hline Malcolmia turkestanica Litw. & Th & IT & & & & \\
\hline Matthiola chenopodifolia Fisch. \& C. A. Mey & Th & IT & & & & \\
\hline Matthiola dumulosa Boiss. \& Buhse. & $\mathrm{Ch}$ & IT & & & * & LR \\
\hline Octoceras lehmannianum Bunge. & Th & IT & & & & \\
\hline Sameraria armena (L.) Desv. & Th & IT & & & & \\
\hline Sameraria elegans Boiss. & Th & IT & & & * & DD \\
\hline Sinapis arvensis L. & Th & $\begin{array}{l}\text { ES-IT- } \\
\text { M-SS }\end{array}$ & * & * & & \\
\hline Sisymbrium septolatum DC. & Th & IT-SS & & & & \\
\hline Sterigmostemum acanthocarpum Fish. \& C. A. Mey. & Th & IT & & & & \\
\hline Sterigmostemum rhodanthum Rech. f. & Th & IT & & & * & DD \\
\hline Tetracme recurvata Bge. & Th & IT & & & & \\
\hline Thlaspi perfoliatum $\mathrm{L}$. & Th & IT & & & & \\
\hline Torularia torulosa (Desf.) O. E. Schulz. & Th & IT-SS & & & & \\
\hline
\end{tabular}


Table 1. Contd.

\begin{tabular}{|c|c|c|c|c|c|c|}
\hline \multicolumn{7}{|l|}{ Capparidaceae } \\
\hline Buhsea trinervia (DC.) Stapf. & G.r & IT & & & & \\
\hline Capparis spinosa L. & $\mathrm{Ch}$ & IT-M-SS & * & * & & \\
\hline \multicolumn{7}{|l|}{ Caryophyllaceae } \\
\hline Acanthophyllum acerosum Sosn. & $\mathrm{Ch}$ & IT & & & & \\
\hline Acanthophyllum crassifolium Boiss. & $\mathrm{Ch}$ & IT & & & & \\
\hline Acanthophyllum glandulosum Bunge ex. Boiss & $\mathrm{Ch}$ & IT & & & & \\
\hline Gypsophila linearifolia (Fisch. \& C. A. Mey.) Boiss. & Th & $\mathrm{IT}$ & & & & \\
\hline Holosteum umbellatum $\mathrm{L}$. & Th & IT-SS & & & & \\
\hline \multicolumn{7}{|l|}{ Chenopodiaceae } \\
\hline Anabasis annua Bge. & Th & IT & * & & & \\
\hline Anabasis setifera Moq. & $\mathrm{Ch}$ & IT-SS & * & * & & \\
\hline Atriplex dimorphostegia var. dimorphostegia Kar \& Kir. & Th & IT-SS & * & & & \\
\hline Atriplex dimorphostegia var. sagitiformis Allen. & Th & IT & * & & & \\
\hline Atriplex leucoclada (Boiss.) Allen. & Hem & IT-SS & * & & & \\
\hline Atriplex moneta Bge. & Th & IT & & & & \\
\hline Atriplex tatarica $\mathrm{L}$. & Th & IT-M & & & & \\
\hline Atriplex verrucifera M. B. & Hem & IT & * & & & \\
\hline Ceratocarpus arenarius $\mathrm{L}$. & Th & IT & * & & & \\
\hline Gamanthus gamocarpus (Moq.) Bge. & Th & IT & * & & & \\
\hline Girgensohnia oppositiflora (Pall.) Fenzel in Ledb. & Th & IT & & & & \\
\hline Halimocnemis mamamensis (Bge.) Assadi, comb. nov. & Th & IT-ES & & & & DD \\
\hline Halimocnemis pilifera Moq. & Th & IT & & & & \\
\hline Halocharis sulphurea Moq. & Th & IT & * & & & \\
\hline Halocharis violaceus Bge. & Th & IT & & & & \\
\hline Halocnemum strobilaceum (Pall.) M. B. & $\mathrm{Ch}$ & IT-SS-M & * & & & \\
\hline Halostachys belangeriana (Moq.) Botsch. & $\mathrm{Ph}$ & IT & * & & & \\
\hline $\begin{array}{l}\text { Halothamnus glaucus subsp. cinerascens (Moq.) } \\
\text { Assadi. }\end{array}$ & $\mathrm{Ch}$ & IT & & & & LR \\
\hline $\begin{array}{l}\text { Halothamnus glaucus subsp. vestitus (Allen.) Assadi, } \\
\text { comb. nov. }\end{array}$ & $\mathrm{Ch}$ & IT & & & & LR \\
\hline Halothamnus subaphyllus Botsch. & $\mathrm{Ph}$ & IT & & & & \\
\hline Haloxylon ammodendron (C. A. Mey.) Bge. & $\mathrm{Ph}$ & IT & * & & & \\
\hline Haloxylon persicum Bge. \& Boiss. et Buhse. & $\mathrm{Ph}$ & IT-SS & * & & & \\
\hline Horaninowia anomala (C. A. Mey.) Moq. & Th & IT & & & & \\
\hline Kalidium caspicum (L.) Ung-Sterb. & $\mathrm{Ch}$ & IT & * & & & \\
\hline Kalidium foliatum (Pall.) Moq. & $\mathrm{Ch}$ & IT & & & & \\
\hline Kochia stellaris Moq. & Th & IT & * & & & \\
\hline Petrosimonia glauca (Pall.) Bge. & Th & IT & & & & \\
\hline Salsola arbuscula Pall. & $\mathrm{Ph}$ & IT & & & & \\
\hline Salsola arbusculiformis Drob. & $\mathrm{Ph}$ & IT & & & & \\
\hline Salsola crasaa M. B. & Th & IT & * & & & \\
\hline Salsola dendroides Pall. & $\mathrm{Ch}$ & IT & * & & & \\
\hline Salsola kali L. & Th & IT & * & * & & \\
\hline Salsola kerneri (Wol.) Botsch. & $\mathrm{Ch}$ & IT & & & & \\
\hline Salsola orientalis S. Gmelin. & $\mathrm{Ch}$ & IT & * & & & \\
\hline Salsola tomentosa (Moq.) Spach. & $\mathrm{Ch}$ & IT & & & & \\
\hline Salsola turcomanica litw. & Th & IT-SS & * & & & \\
\hline Seidlitzia florida (M. B.) Boiss. & Th & IT & * & & & \\
\hline Seidlitzia rosmarinus (Ehrh.) Bge. & $\mathrm{Ph}$ & IT-SS & * & & & \\
\hline
\end{tabular}


Table 1. Contd.

\begin{tabular}{llc}
\hline Suaeda acuminata (C. A. Mey.) Moq. & Th & IT \\
Suaeda microphylla Pall. & Ph IT-ES \\
Suaeda microsperma (C. A. Mey.) Fenzel in Ledeb. & Th IT & \\
& & \\
Convolvulaceae & Ch IT-SS \\
Convolvulus eremophilus Boiss. et Buhse. & Hem IT-SS * \\
Convolvulus pilosellaefolius Desr. & Hem IT-M-SS \\
Cressa cretica L. & * DD
\end{tabular}

\section{Cyperaceae}

Carex physodes Bieb.

G.r IT

Scirpus maritimus L.

G.r IT

Dipsaceae

Scabiosa olivieri Coult.

Scabiosa rotata M. B.

Th

IT-SS-ES

\section{Ephedraceae}

Th

IT

Ephedra sarcocarpa Aitch. et Hemsl.

Ephedra strobilacea Bge. ex Lehm.

\section{Euphorbiaceae}

Chrozophora tinctoria (L.) Juss.

Euphorbia cheirolepioides Rech. f.

Euphorbia densa Schrenk.

Euphorbia heteradenia laub. \& Spach.

Euphorbia sororia Schrenk

Euphorbia turcomanica Boiss.

$\mathrm{Ph}$

$\mathrm{Ph}$

Th

Th

Th

Hem

Th

Th

Fumariaceae

Fumaria parviflora Lam.

Geraniaceae

Erodium oxyrrhynchum M. B.

Iridaceae

Iris kopetdaghensis (Vved.) Mathew \& Wendelbo.

Iris songarica Schrenk

\section{Ixioliriaceae}

Ixiolirion tataricum Fisch. ex Herb.

\section{Lamiaceae}

Eremostachys hyoscyamoides Boiss \& Buhse.

Lallemantia royleana (Benth. In Walt.) Benth.

Salvia reuterana Boiss.

Stachys trinervis Aitch \&Hemsl.

Thuspeinanta persica (Boiss.) Briq.

Ziziphora tenuir L.
Th

Th

G. b

G. $r$

G. b IT-SS-ES *

Liliaceae

Allium borszczowii Regel.

G. b IT 
Table 1. Contd.

\begin{tabular}{|c|c|c|c|c|c|c|}
\hline Allium capsicum (Pall.) M. B. & G. b & IT & & & & \\
\hline Eremorus inderiensis Stev.)Boiss. & G. $t$ & IT & & & & \\
\hline Gagea reticulata (Pall.) Schultes & G. b & IT & & & & \\
\hline Tulipa biflora Pall. & G. b & IT & & & & \\
\hline Tulipa montana Lindl. var.montana & G. b & IT & & * & * & DD \\
\hline \multicolumn{7}{|l|}{ Orobanchaceae } \\
\hline Orobanch picridis FW. Schultz. & Par & IT & & & & \\
\hline \multicolumn{7}{|l|}{ Papaveraceae } \\
\hline Glaucium elegans Fisch. \& C. A. Mey. & Th & IT & & & & \\
\hline Hypecoum pendulum L. & Th & IT-M-SS & * & & & \\
\hline Romeria hybrida (L.) DC. & Th & IT-SS & & & & \\
\hline \multicolumn{7}{|l|}{ Papilionaceae } \\
\hline Alhagi persarum Boiss. \& Buhse. & Hem & IT & * & * & & \\
\hline Astragalus angustatus Boiss. & Hem & IT & * & & * & LR \\
\hline Astragalus argyroides G. Beck. & Hem & IT & * & & & \\
\hline Astragalus arpilobus (Boiss.) Podl. & Th & IT-SS & & & & \\
\hline Astragalus bakaliensis Bunge. & Th & IT & & & & \\
\hline Astragalus campylorrhyncus F. \& M. & Th & IT & & & & \\
\hline Astragalus commixtus Bunge & Th & IT & & & & \\
\hline Astragaluscorronilla Gazer \& Podl. & Th & IT & & & & \\
\hline Astragalus crenatus Schultes & Th & IT-SS & & & & \\
\hline Astragalus dactylocarpus Emend. Ott. & $\mathrm{Ch}$ & IT & & & & \\
\hline $\begin{array}{l}\text { Astragalus eremophilus subsp. eremophilus Emend. } \\
\text { Podlech }\end{array}$ & Th & IT-SS & & & & \\
\hline Astragalus eremophilus subsp. makranicus Podlech & Th & IT-SS & & & & \\
\hline Astragalus kahiricus DC. & Hem & IT-SS & & * & & \\
\hline Astragalus macrobotrys Bunge & $\mathrm{Ch}$ & IT & & & & \\
\hline Astragalus nigricans Barneby & $P$ & IT & & & & \\
\hline Astragalus oxyglottis Bieb. & Th & IT & & & & \\
\hline Astragalus pellitus Bunge & Hem & IT & & & & \\
\hline Astragalus podolobus Boiss. \& Hohen. & $\mathrm{Ch}$ & IT & & & & \\
\hline Astragalus tribuloides Delile & Th & IT-SS & & & & \\
\hline Ophiocarpus sp. & Th & IT & & & & \\
\hline Sophora pachycarpa C. A. Mey. & Hem & IT & & & & \\
\hline Trigonella calliceras Fisch. & Th & IT & * & & & \\
\hline \multicolumn{7}{|l|}{ Plantaginaceae } \\
\hline Plantago evacina Boiss. & Th & IT & & & * & \\
\hline \multicolumn{7}{|l|}{ Plumbaginaceae } \\
\hline Acantholimon acmostegium Boiss. \& Buhse. & $\mathrm{Ch}$ & IT & & & * & LR \\
\hline Acantholimon scorpius (Jaub. \& Spach) & $\mathrm{Ch}$ & IT & & & * & LR \\
\hline Acantholimon acerosum (Wild.) Boiss. & $\mathrm{Ch}$ & IT & & & & \\
\hline \multicolumn{7}{|l|}{ Poaceae } \\
\hline Aeluropus littoralis (Gouan) Parl. & G. $r$ & IT-M-SS & * & & & \\
\hline Boissiera squarrosa Hochst. ex Steud. & Th & IT & * & & & \\
\hline Bromus danthoniae Trin. & Th & IT & * & & & \\
\hline Bromus sericeus Drobv. & Th & IT & & & & \\
\hline
\end{tabular}


Table 1. Contd.

\begin{tabular}{|c|c|c|c|c|c|c|}
\hline Bromus tectorum $\mathrm{L}$. & Th & Cosm & * & * & & \\
\hline Eremopyrum bonaepartis (Speng.) Nevski & Th & IT & * & & & \\
\hline Eremopyrum distans (C. Koch.) Nevski & Th & IT & & & & \\
\hline Hordeum glaucum Steud. & Th & IT-M & * & & & \\
\hline Nardurus subulatus (Banks \& Soland.) Bor. & Th & IT & & & & \\
\hline Phalaris minor Retz. & Th & IT-M & * & & & \\
\hline Poa bulbosa L. & G. b & IT-M-ES & * & & & \\
\hline Schismus arabicus Nees. & Th & IT-M-SS & & & & \\
\hline Stipa caucasica Schmalh. & Hem & IT-ES & & & & \\
\hline Stipa lessingiana Trin. \& Rupr. & Hem & IT-Es & * & & & \\
\hline Stipagrostis pennata (Trin.) De. Winter & G. $r$ & IT & * & & & \\
\hline Stipagrostis plumosa (L.) Munro. ex. T. Anders & Hem & IT-SS & * & & & \\
\hline \multicolumn{7}{|l|}{ Polygonaceae } \\
\hline Atraphaxis spinosa L. & $\mathrm{Ph}$ & IT-M & & * & & \\
\hline Calligonum denticulatum Bge. ex Boiss. & $\mathrm{Ph}$ & $\mathrm{IT}$ & & & * & \\
\hline Calligonum junceum (Fisch. \&C.A.Mey.) Litw. & $\mathrm{Ph}$ & IT & & & & \\
\hline Polygonum hyrcanicum Rech. f. & Th & IT-ES & & & * & LR \\
\hline Polygonum olivascens Rech. f. \& Schiman- Czeika & Th & IT-SS & & & * & \\
\hline Polygonum patulum M. B. & Th & IT-M & & & & \\
\hline Pteropyrum aucheri Jaub. \& Spach. & $\mathrm{Ph}$ & IT & * & & * & \\
\hline \multicolumn{7}{|l|}{ Primulaceae } \\
\hline Anaghalis arvensis $\mathrm{L}$. & Th & IT-M-ES & * & * & & \\
\hline \multicolumn{7}{|l|}{ Ranunculaceae } \\
\hline Ceratocephalus falcatus (L.) Pers. & Th & IT-M & & & & \\
\hline Consolida rugulosa (Boiss.) Schrod. & Th & $\mathrm{IT}$ & & & & \\
\hline \multicolumn{7}{|l|}{ Resedaceae } \\
\hline Reseda buhseana Mull-Arg. var. buhseana Mull- Arg. & Hem & IT & & & & \\
\hline \multicolumn{7}{|l|}{ Rosaceae } \\
\hline Rosa persica Michx.ex Juss. & $\mathrm{Ph}$ & IT & & & & \\
\hline \multicolumn{7}{|l|}{ Rubiaceae } \\
\hline Callipeltis cucullaria Stev. & Th & IT & & & & \\
\hline Leptunis trichodes (J. Gay) Schischk & Th & IT & & & & \\
\hline Gaillonia brungieri A. Rich. & G. $r$ & IT-SS & & & & LR \\
\hline \multicolumn{7}{|l|}{ Rutaceae } \\
\hline Haplophyllum glaberrimum Bge. ex Boiss. & $\mathrm{Hem}$ & IT & & & * & LR \\
\hline Haplophyllum sp. & Hem & IT & & & & \\
\hline \multicolumn{7}{|l|}{ Solanaceae } \\
\hline Hyoscyamus pusillus L. & Th & IT & & & & \\
\hline Lycium ruthenicum Murr. & $\mathrm{Ph}$ & IT & & & & \\
\hline \multicolumn{7}{|l|}{ Tamaricaceae } \\
\hline Reaumuria cistoides Adam. & $\mathrm{Ch}$ & IT-ES & & & & \\
\hline $\begin{array}{l}\text { Reaumuria oxiana (Ledeb.) Boiss var. persica (Boiss.) } \\
\text { Assadi }\end{array}$ & $\mathrm{Ch}$ & $\mathrm{IT}$ & & & * & LR \\
\hline
\end{tabular}


Table 1. Contd.

\begin{tabular}{|c|c|c|c|c|c|}
\hline Tamarix gallica L. & $\mathrm{Ph}$ & IT-SS & & * & \\
\hline Tamarix macrocarpa (Ehrenberg.) Bge. & $\mathrm{Ph}$ & IT-SS & & & \\
\hline \multicolumn{6}{|l|}{ Valerianaceae } \\
\hline Valerianella cymbicarpa C. A. Mey. & Th & IT & & & \\
\hline Valerianella dufresnia Bge. ex Boiss. & Th & IT & & & \\
\hline Valerianella oxyrrhynca Fisch \& C. A. Mey. & Th & IT & & & \\
\hline Valerianella szwitsiana Fisch. \& C. A. Mey. & Th & IT & & & \\
\hline Valerianella triplaris Boiss \& Buhse. & Th & IT & & & \\
\hline Valerianella turkestanica Regel \& Schmalh. ex Regel. & Th & IT & & & LR \\
\hline \multicolumn{6}{|l|}{ Zygophyllaceae } \\
\hline Nitraria schoberi L. & $\mathrm{Ph}$ & $\mathrm{IT}$ & * & & \\
\hline Peganum harmala L. var harmala & Hem & IT-M-SS & & * & \\
\hline Tribulus macropterus Boiss. & Th & IT-SS & & & \\
\hline Tribulus terrestris L. var terrestris & Th & IT-SS-ES & & * & \\
\hline Zygophyllum atriplicoides Fisch. \& C. A. Mey. & $\mathrm{Ph}$ & IT-SS & * & & \\
\hline Zygophyllum eurypterum Boiss. \& Buhse. & $\mathrm{Ph}$ & IT-SS-M & & & \\
\hline Zygophyllum miniatum Cham. \& Schlechtend. & Hem & IT & * & & \\
\hline
\end{tabular}

L.F: Life form, Chor: chorotype, Pa: pasture, Me: medicinal, En: endemic, Vu: vulnerability, Th: therophyte; Hem: hemicryptophyte, Ch:Chamaephyte, Ph:phanerophyte, G.t geophyte tuberous, G.b: geophytes bulbous; G.r: geophytes rhizomous, IT: Irano-Turanian, M :Mediterranian, SS: Sahara-Sindian, ES: Europa-Siberian, DD: data deficiency, LR: lowrisk, Par: parasite.

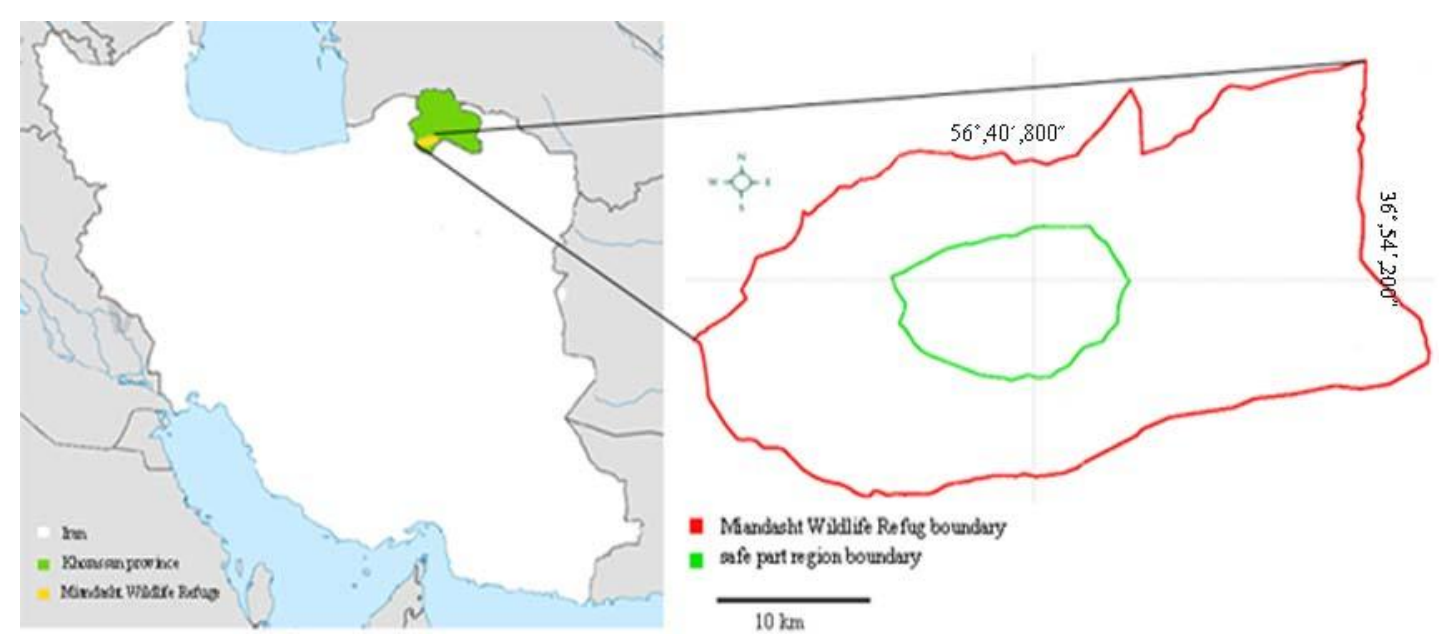

Figure 1. Location of Miandasht Wildlife Refuge on Iran map (drawn using Adobe Photoshop CS4).

this region (Maassoumi, 1986-2005). Hence, the maximum number of Astragalus species was expected in Miandasht because it is situated in the Irano-Turanian region.

The floristic composition of the vegetation expressed the climatical and edaphical conditions of this region. In this composition, relatively high presence of certain groups of plants, that each of them grows in specific environmental conditions, could be observed. These groups include the desert plants (Artemisia sieberi, Cousinia neurocentra, Heliotropium aucheri, Acantholimon crassifolium), psammophytes (Carex physodes, Calligonum junceum, Schomannia karelinii, Psammogeton canescens), desert halophytes (Anabasis annua, Atriplex moneta, atriplex dimorphostegia, Halimocnemis pilifera, Halocharis sulphurea, Seidlitzia rosmarinus, Salsola turcomanica), marsh halophytes (Kalidium capsicum, Halocnemum strobilaceum, 


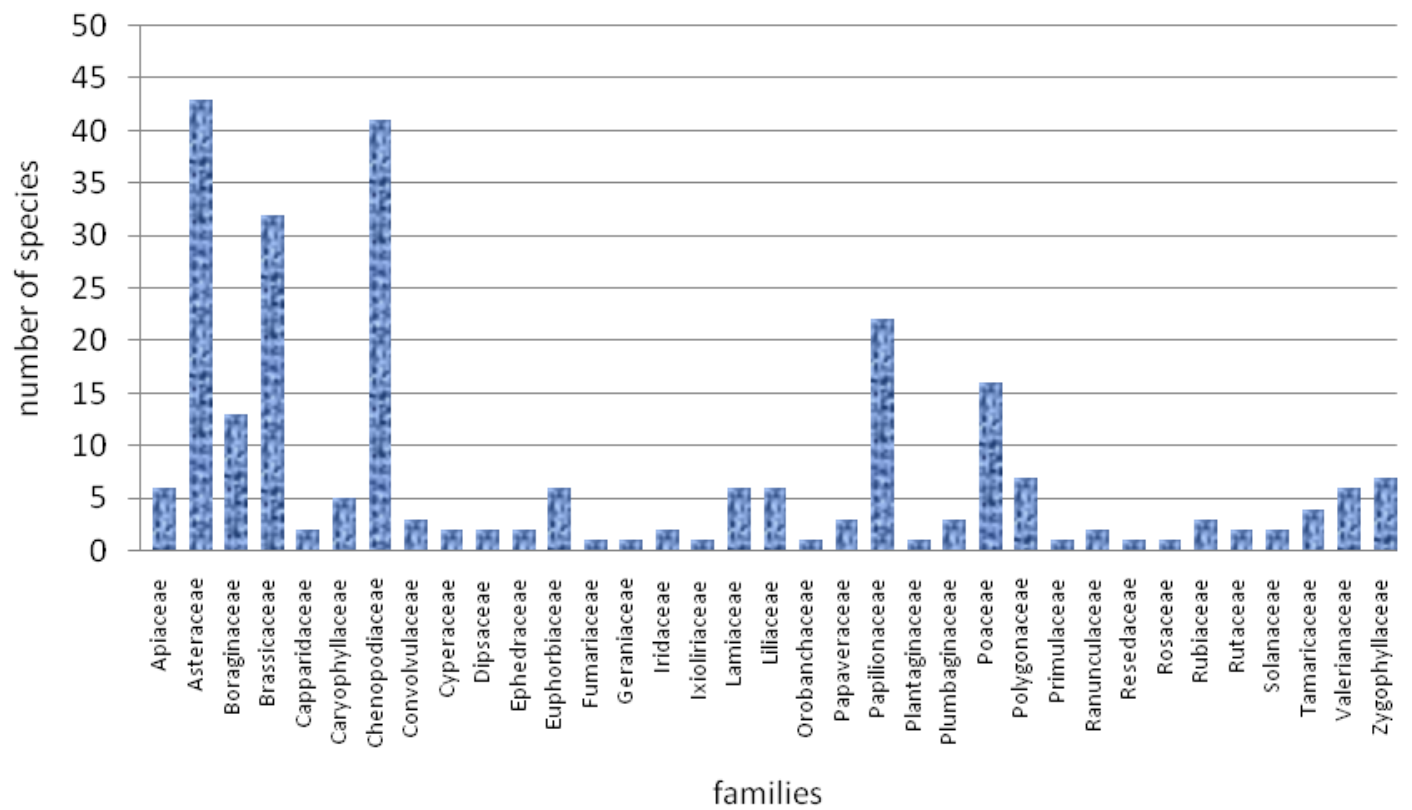

Figure 2. Chart of the number of species belonging to families in flora of Miandasht Wildlife Refuge (out of the safe part).

Aeloropus littoralis, Phragmites australis) and a large number of ruderals, for example: Buhsea trinervia, Cardaria draba, Launaea acanthodes, Peganum harmala, Sophora Pachycarpa, Acantholepis orientalis. Thelarge number of annual and perennial anthropogenicand ruderal plants in this area indicate excessive degradation, especially as a result of overgrazing and undermining by livestock. If rangelands are allowed to regenerate properly and grazing controlled in selected area, conservation can be achieved and the long term stability of the pastural life-style can be enhanced (Shahina and Ghazanfar, 1998).

Figure 3 illustrates that the therophytes with 143 species $(55.86 \%)$ were the maximum number of life form on the flora of this area, then there were 42 (15.62) hemicryptophytes, $26(10.16 \%)$ chamephytes, 23 (8.6\%) phanerophytes and totally $20(9.38 \%)$ geophytes.

The Orobanch picridis is a parasitic species that grows in this area. Among the geophytes, $8(3.125 \%)$ rhizomous species, $8(3.125 \%)$ tuberous species and $8(3.125 \%)$ bulbous species were found. Therophytes are reproduced by the seeds. Compatibility of this procedure for reproduction in arid areas is more than other ways, because seeds are reproduced in small size and large number. So, they are distributed very easily. Seeds usually survived on unfavorable conditions. Also, genetic diversity of seeds which are produced through sexual reproduction leads to genetic flexible populations (Neishabouri, 1995). Most of the therophytes that grow in MWR are adapted with short duration of precipitation and high temperature, and they complete the life cycle and produce the seeds. Saberamoli (2001) said that when the numbers of therophytes exceed the other life forms it shows desert conditions.

According to Table 1, 29 (11.33\%) Iran endemic species grow in MWR. The presence of endemic species is among the fundamental criteria for characterizing biodiversity of a territory (Giuseppe, 2013). On the other hand, there are no obvious correlation between modern climate and endemism (Linder, 2001). Iran is one of the main centers of endemism in the world (Saberamoli, 2001). Totally 2000 endemic species grow in Iran and Irano-Turanian region contain $85 \%$ of endemic plant species of Iran (Dehshiri, 2005). The results of this research show that in the MWR which is located in IranoTuranian region grow $1.45 \%$ of all endemic species of Iran. Since extent of this area is much less than the Irano-Turanian region in Iran, the above result was justifiable. Chamephytes, for example Artemisia and some species of salsola, are relatively big shrubs and their production is more than that of therophytes. Also, they are significant component of vegetation in a long period of the year. By increasing drought trend from spring to late summer, also by decreasing temperature until autumn, dominance of the chenopodiaceae in the plant formation of the study area was clearly evident. They are resistant to salinity and drought. Therefore, they grow very well in spite of intense environmental conditions (Saberamoli, 2001). Chamephytes and Phanerophytes are resistant to drought and they are morphologically adapted instead of adaptation in life cycle (Saberamoli, 2001). Although these two groups were not large in the life form spectrum of MWR, they were the main component of vegetation. Also, most of them are 


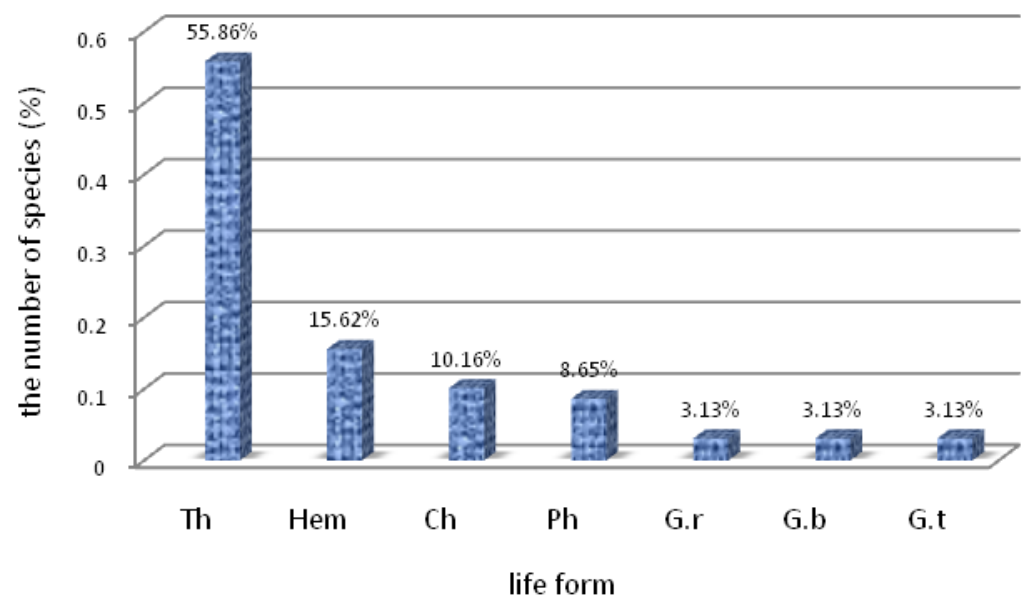

Figure 3. Life form spectrum of flora of Miandasht Wildlife Refuge (out of the safe part). Th: therophyte, Hem: hemicryptophyte, Ch: chamephyte, Ph: phanerophyte, G.r: geophyte with rhizome, G.b: geophyte with bulb, G.t: geophyte with tuber.

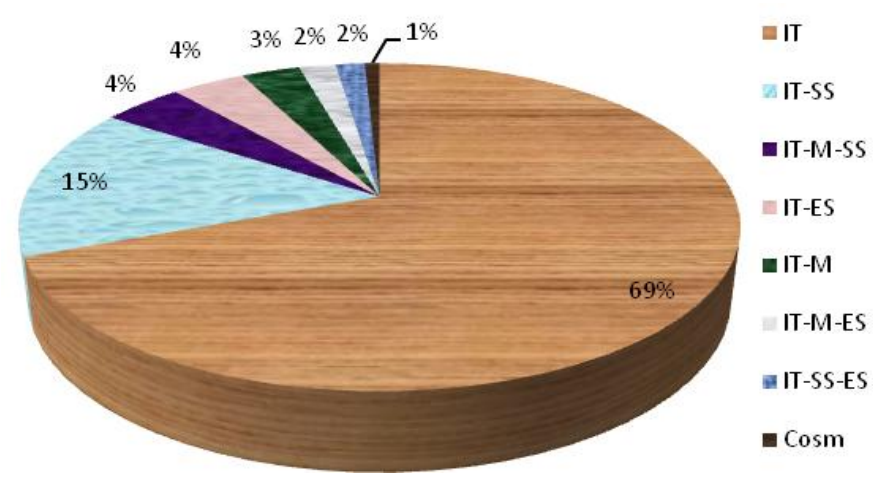

Figure 4. Weight of chorotype on flora of Miandasht Wildlife Refuge (out of the safe part).

effective in preventing erosion. In addition to these, majority of them are pasture plants and provide food for wildlife. Therefore, these facts should be considered in management of this area. Because geophytes have underground organs like tubers, bulbs and rhizomes, they need suitable soil depth. Relatively high presence of geophytes showed that this area had capability of pedogenes.

Figure 4 show that the majority of species were IranoTuranian elements. The remaining plants were common elements between Irano-Turanian and other regions. Ascending order were Irano-Turanian and SaharaSindian, Irano-Turanian and Euro-Siberian, IranoTuranian and Mediterranian and Sahara-Sindian, IranoTuranian and Euro-Siberian, Irano-Turanian and Mediterranian, Irano-Turanian and Mediterranian and Euro-Siberian, and Cosmopolitan and Irano-Turanian and Sahara-Sindianand Euro-Siberian.

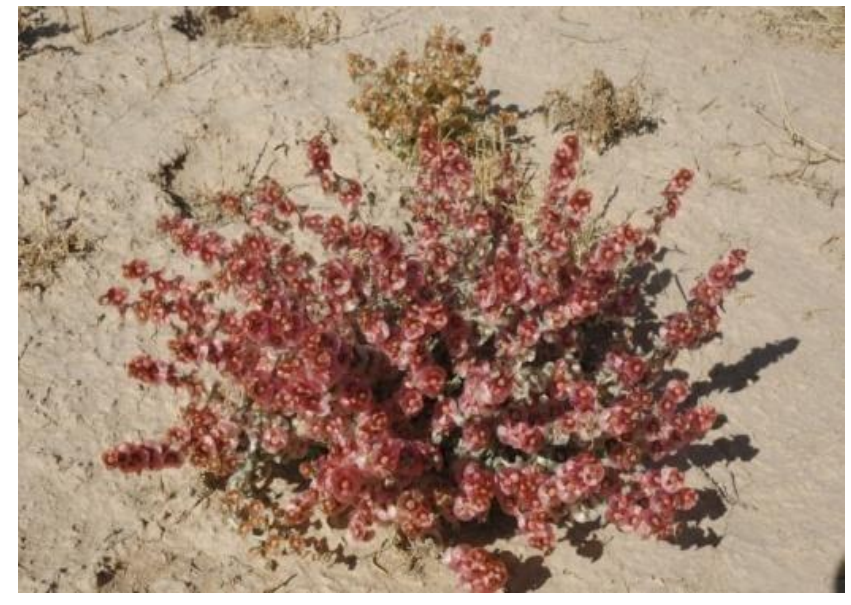

Figure 5. Salsola turcomanica.Litw.

Table 1 shows that from the 256 species, 64 were pasture plants and 38 were medicinal. Shrubs like Artemisia, especially different shrub and herbal Chenopodiaceae species have high forage value. They were widely distributed in this area, and they had high production. Therefore, MWR is a good pasture.

As the number of rare and threatened species has multiplied, it has become increasingly important to select species for conservation management and to provide information on the causes of decline (Perrine et al., 2013). Fortunately, this study showed that any of the species existing in MWR were not at risk or vulnerable. The current picture of extinction risk is still incomplete because many species in flora of MWR were never assessed.

We concluded on the plant diversity of MWR, in spite of environmental stresses such as drought, salinity, erosion 


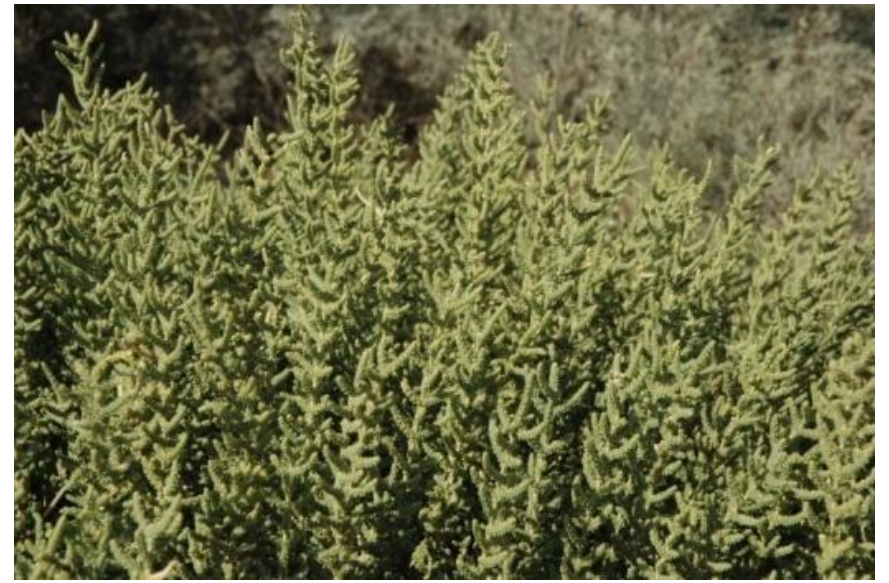

Figure 6. Halocnemum strobilaceum (Pall)M. B.

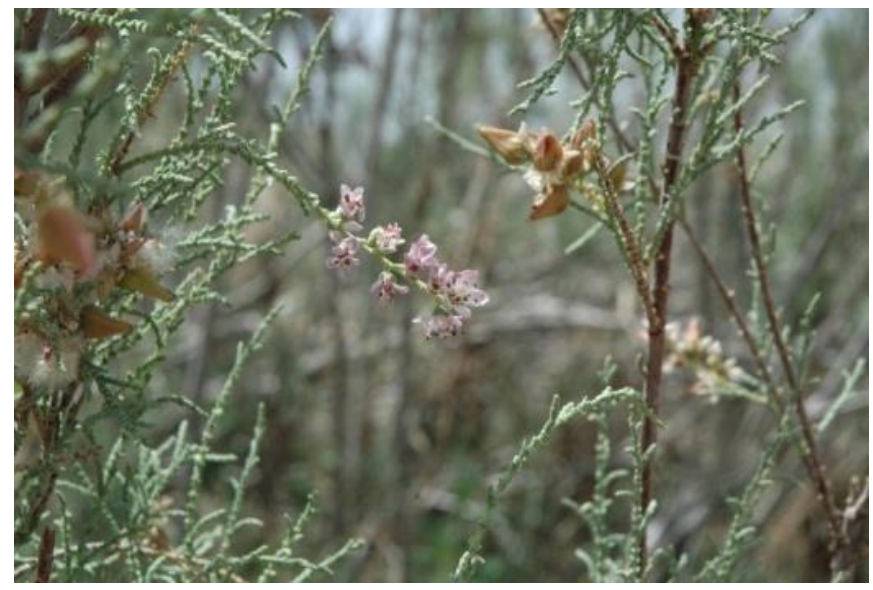

Figure 8. Tamarix macrocarpa (Ehrenberg.) Bge.

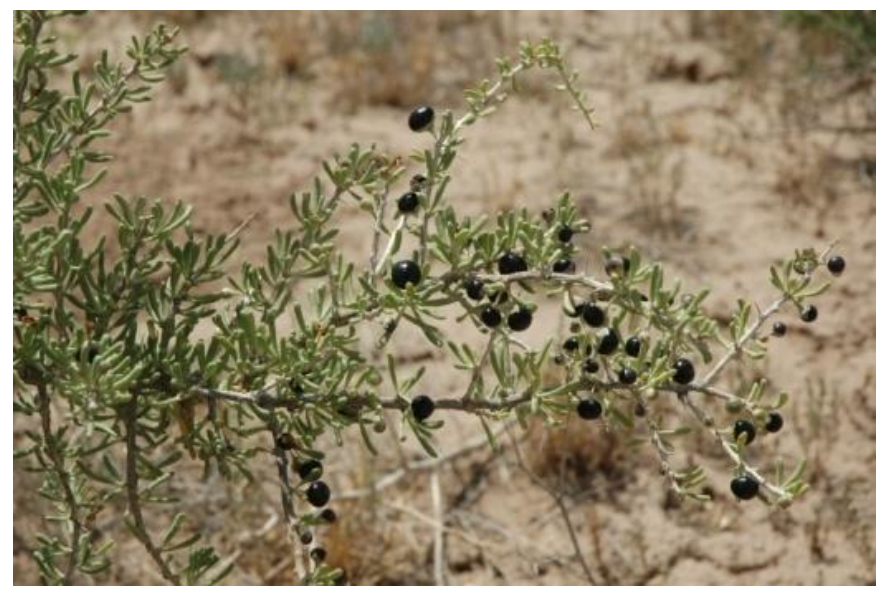

Figure 10. Lycium ruthenicum Murr.

and uncontrolled grazing by livestock is high. Moreover, this diversity is manifested not only in number of species

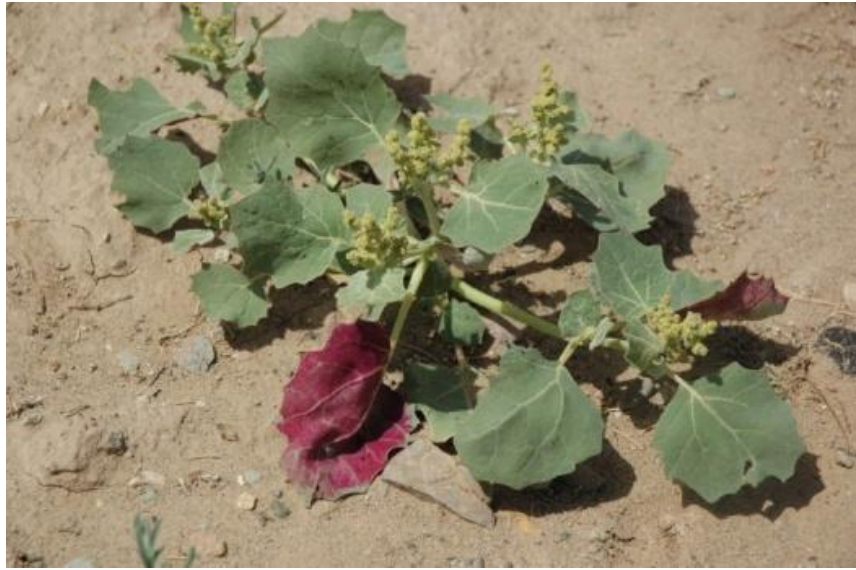

Figure 7. Atriplex moneta Bge.

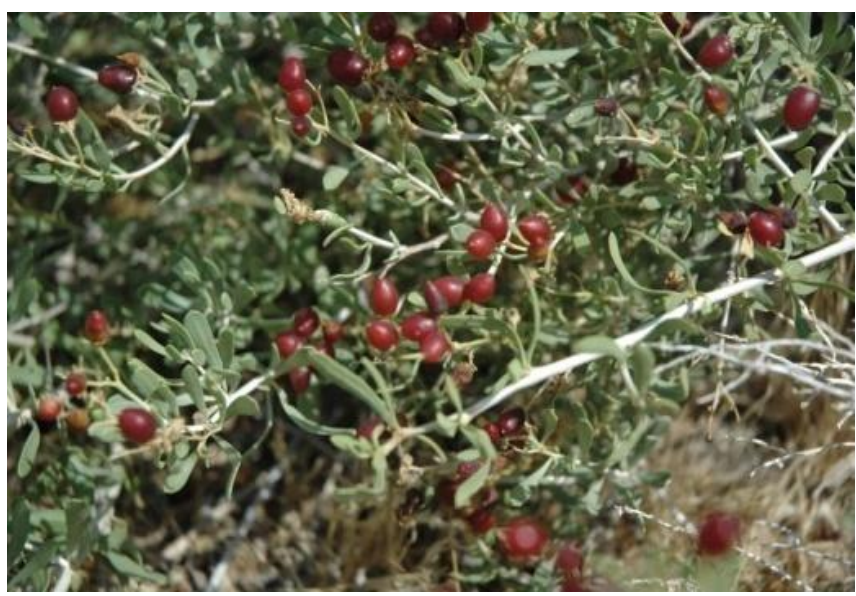

Figure 9. Nitraria schoberi L.

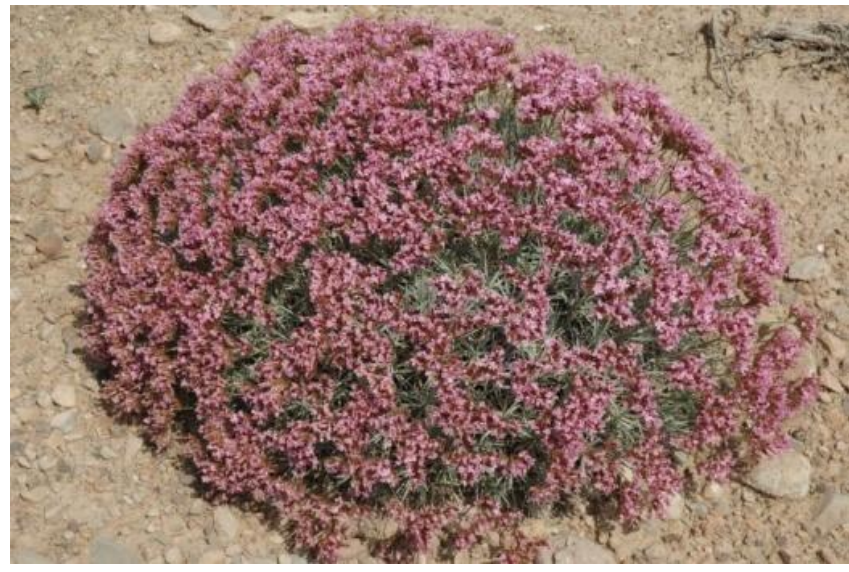

Figure 11. Acantholimon acmostegium Boiss. \& Buhse.

but also in terms of the chorotype and life-form presents. In this region, the diversity of microclimates and the 


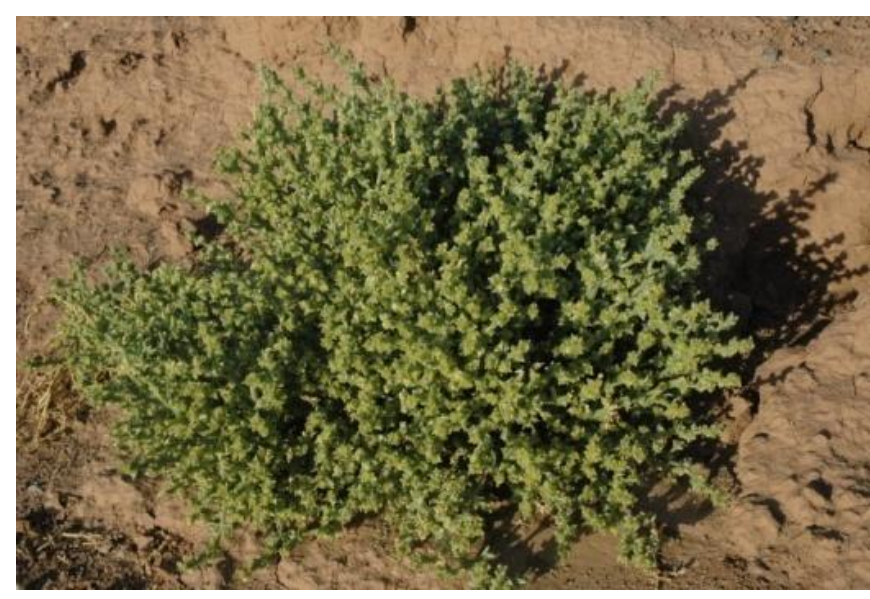

Figure 12. Anabasis setifera Moq.

physiographic units of dry and saline areas as hillsides plains, sandy deserts, seasonal streamlets and Kavir areas have made many different local edaphic conditions.

Among the plants, Chenopodiaceae species are very important for providing the food for wildlife. Specially, the shrubs which have large amounts of proteins and minerals are important. These plants are a valuable source of food for wildlife. Overall, the flora consists of plants that have adapted to climatic and edaphic conditions of the region in life-form or morphology or life cycle. Also, many plants are invasive. These plants are distributed in this area due to the natural erosion and degradation resulting from human interventions. So, proper management and protection of the existing sources can increase species richness.

\section{ACKNOWLEDGEMENTS}

This study was funded by Islamic Azad University, Bojnourd Branch. We are thankful to the Northern Khorassan Provincial Directorate of Environment Protection and Jajarm Department of Environmental Protection for providing necessary facilities during the field surveys. We would like to thank Dr. Maassoumi and Dr. Mozaffarian for helping us in identifying the plants.

\section{REFERENCES}

Akhani $\mathrm{H}$ (1989). A contribution to the vegetation and flora of kavire Meyghan (NE.Arak), Iran. J. Sci.Univ. Tehran 18:75-84.

Akhani H (2006). Flora Iranica, Facts and Figures and a List of Publications by K.H. Rechinger on Iran and adjacent areas. Rostaniha 7 (suppl.2).

Amin Gh (1991). Medicinal plant of Iran. Ministry of Health, Therapy and Medical teaching press, Tehran.

Asri Y (2003). Plant diversity in Kavir biosphere reserve. Research Institute of Forests and Rangelands press,Tehran.

Asri Y (2007). Flora of halophytes of Iran. The proceeding of 1st national Plant Taxonomy Conference of Iran. Research Institute of Forests and Rangelands, Tehran.
Assadi M (1984). Studies on the autumn plants of kavir, Iran. Iran. J. Bot. 2 (2): $125-148$.

Assadi $M$ et al (1988-2011). Flora of Iran. Vol: 1-71 Research Institute of Forests and Rangelands press,Tehran.

Boissier E (1936). Flora Orientalis. Genevae \& Basileae, Lugduni.

Bornmuller J, Gauba E (1935 -1940). Flora keredjensis Fundamenta. Cambridge University Press, Cambridge.

David JC, Kenneth AA (2001).Prioritysetting and the conservation of Western Australia's diverse and highly endemic flora. Biol. Conserv. 97(2): 251-263.

Davis PH (1965 - 1985). Flora of Turkey and the East Aegean Islands. Vols 1-9 Edinburg Univ. Press,Edinburgh.

Dehshiri MM (2005). Study of plant associations of Dizin, Gagereh and Velayatrood regions.Dessertation, Science and Research branch of Islamic Azad University of Tehran.

Freitag $H$ (1986). Notes on the distribution, climate and flora of the sand deserts of Iran and Afghanistan. Proceeding of the Royal Society of Edinburg. Edinburg.

Ghahreman A (1977-2007). Colored flora of Iran.Vols 1-26, Research Institute of Forests \& Rangelands press, Tehran.

Giuseppe B (2013). Adaptive management as a tool to improve the conservation of endemic floras:the case of Sicily, Malta and their satellite islands. Biodivers. Conserv. 22(6-7): 1317-1354.

Javidtalesh I (2001). Medicinal plant of Fars province. Res. Med. Aromat. Plants 11: 103-148.

Komarov VL (1934-1957). Flora of U. S. S. R. Vols 1-30, The Botanical Institute of science of the U.S.S.R., Leningrad.

Linder HP (2001). Plant diversity and endemism in sub-saharian tropical Africa. J. Biogeogr. 28(2): 169-182.

Maassoumi AA (1986-2005). The genus Astragalus in Iran. Vols 1-5, Research Institute of Forests \& Rangelands, Tehran.

Mobayen S (1975-1995). Flora of Iran, Teracheophyta Flora. Vols 1-4, Tehran University.Tehran.

Mozaffarian V (2007). Plant distribution In Iran and endemism in Iran The proceedings of 1st national Plant Taxonomy conference of Iran. Tehran.

Nasir E, Qaiser M (1970-2001). Flora of West Pakistan.Vols 1-202, B.C.C. and T Press, University of Karachi.

Neishabouri A (1995). Biogeography. Study and Collection of human science books organization. Samt, Tehran.

Parsa A (1948-1952). Flore de l'Iran. Offset Press Ink.

Perrine G, Yoann, Olivier J, John DT (2013).Quantifying habitate vulnerability to assess species priorities for conservation management. Biol. Conserv.158:321-325.

Raunkaier C (1934). Plant Life Forms. Clarendon Press, Oxford.

Rechinger KH, Wendelbo P (1976). Plants of the Kavir Protected Area, Iran. Iran. J. Bot. 1: 23-56.

Rechinger KH (1963 - 2005). Flora Iranica. Nos 1-176, Akademische Druck velsanstalt, Graz - Aust.

Rechinger KH (1977). Plants of the Touran Protected Area, Iran. Iran. J. Bot. 1(2):155 - 180.

Saberamoli S (2001). Floristic study and preparation of vegetation map of Mahroieh wildlife refuge of Kerman. Dissertation, Tarbiat Moallem Univ.press , Tehran.

Shahina A, Ghazanfar (1998).Status of the flora and plant conservation in the Sultanate of Oman. Biol. conserv.85: 287-295.

Takhtajan A (1986). Floristic Regions of the World.University of California Press, California.

Tavakkoli Z, Mozaffarian V (2005). Study of the flora of Kabar dam of Ghom. Pajouhesh and Sazandegi J. 17 (1): 22-29.

Townsend CC, Guest E (1966-1986). Flora of Iraq.Vols 1-9 Ministry of Agriculture and Agrarian Reform, Baghdad.

Turan Y, Filiz Y (2011). The effects of restoration on soil properties in degraded land in the semi-arid region of turkey. Catena 84(1-2) 4753.

Yousofinajafabadi M (1996). The study of flora and preparation of plant vegetation of Ghomeshlou preserved region. Dissertation, Shahid Beheshti University,Tehran.

Zargary A (1999). Medicinal plants. Vol 1-5, Tehran University Press,

Tehran.

Zohary M (1973), Geobotanical Foundation of the Middle East. 2 Vols, Gustav Fischer Verlag, Stuttgart. 